\title{
Genes encoding hub and bottleneck enzymes of the Arabidopsis metabolic network preferentially retain homeologs through whole genome duplication
}

\author{
Xudong Wu and Xiaoquan Qi*
}

\begin{abstract}
Background: Whole genome duplication (WGD) occurs widely in angiosperm evolution. It raises the intriguing question of how interacting networks of genes cope with this dramatic evolutionary event.

Results: In study of the Arabidopsis metabolic network, we assigned each enzyme (node) with topological centralities (in-degree, out-degree and between-ness) to measure quantitatively their centralities in the network. The Arabidopsis metabolic network is highly modular and separated into 11 interconnected modules, which correspond well to the functional metabolic pathways. The enzymes with higher in-out degree and between-ness (defined as hub and bottleneck enzymes, respectively) tend to be more conserved and preferentially retain homeologs after WGD. Moreover, the simultaneous retention of homeologs encoding enzymes which catalyze consecutive steps in a pathway is highly favored and easily achieved, and enzyme-enzyme interactions contribute to the retention of onethird of WGD enzymes.
\end{abstract}

Conclusions: Our analyses indicate that the hub and bottleneck enzymes of metabolic network obtain great benefits from WGD, and this event grants clear evolutionary advantages in adaptation to different environments.

\section{Background}

Whole genome duplication is one of the most important evolutionary events in plants [1] and many duplicated genes retained as large blocks have been found in the Arabidopsis [2], rice [3,4] and Populus [5] genomes. In Arabidopsis, a genome-wide similarities search (Blast) was done among protein-coding genes and strong evidence for a whole genome duplication event was demonstrated by phylogenetic analysis. The analysis of the genomic duplication blocks revealed that about 80 percent of genes lost their sister genes following WGD $[2,6]$. In rice, analysis using the structural genomic data and phylogenetic analysis suggested that a polyploidization event occurred about 50 70 million years ago, which was before the divergence of the major cereals but after the divergence of the Poales from the Liliales and Zingiber-

\footnotetext{
*Correspondence: xqi@ibcas.ac.cn

${ }^{1}$ Key Laboratory of Photosynthesis and Environmental Molecular Physiology, the Institute of Botany, Chinese Academy of Sciences, Beijing 100093, China Full list of author information is available at the end of the article
}

ales [3,4]. Analysis of the assembled Populus genome sequences revealed evidence for a whole-genome duplication event in the genome, where about 8000 pairs of homeologs survived after the event [5].

Research on the genome data from vertebrates and yeast has shown that factors, such as gene expression intensity [7,8], protein interaction [9], phylogenetic age [10], and dosage sensitivity [11] have influenced the evolutionary rates or the retention of homeologs after gene duplication. Recent research on the unicellular ciliate protozoa, Paramecium [12], showed that metabolic genes appear more retained than other types of genes after WGD. In Arabidopsis, dosage effects were suggested to be an important factor influencing the retention of homeologs. For example, transcription factors, which have roles in regulating other genes, normally show strong dosage sensitivity and genes encoding transcription factors were over-represented amongst WGD-homeologs as shown by GO analysis [11]. Although plants have evolved the ability to synthesize a vast array of metabolites which 
are essential for adaptation to diverse natural environments [13-15], the evolution of plant metabolic networks has not been studied extensively. It is of particular interest to know how plant metabolic networks cope with whole genome duplication events.

Graph theory provides paradigms to study networks [16]. The plant metabolic network is the well-known biological network [17]. Its enzymes can be represented by nodes and substrate-product metabolite flux can be represented by directional edges (as demonstrated in Figure 1A). Three topological centralities are used to measure the importance of nodes in the control of information transfer. In-degree refers to the number of links forwarded to the considered nodes, out-degree refers to the number of links outwards from the considered nodes and between-ness measures the propensity of shortest paths from any other nodes going through a certain node. In Figure 1B, five enzymes provide products for node $\alpha$, which produces one product for the next biosynthesis step (node $\beta$ ), so the in-degree of node $\alpha$ is assigned by 5 whereas its out-degree by 1 . Node $\alpha$ and node $\beta$ are the essential nodes for successful information transfer from the blue nodes to the yellow nodes, if either of them is knocked out, the network would collapse. Obviously, in-

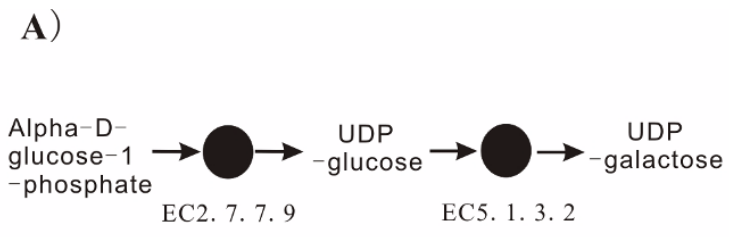

C)

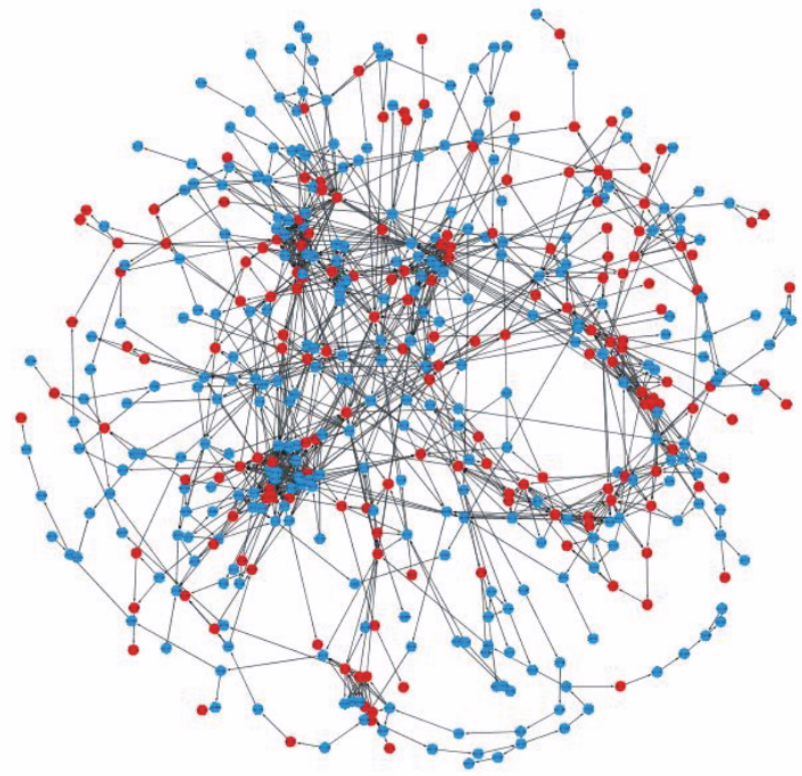

B)

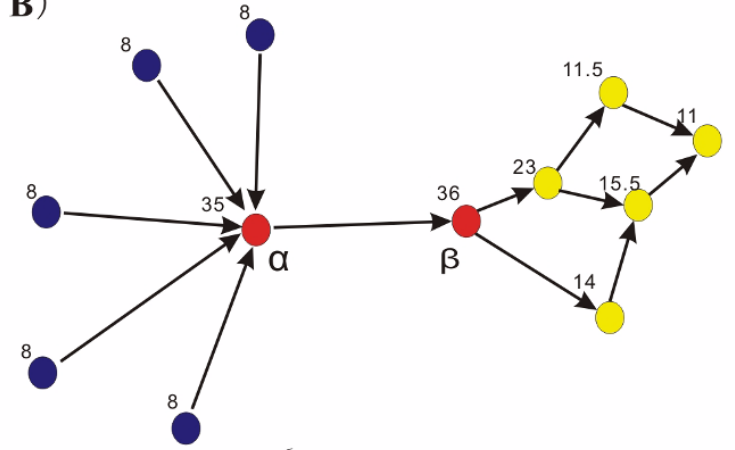

D)

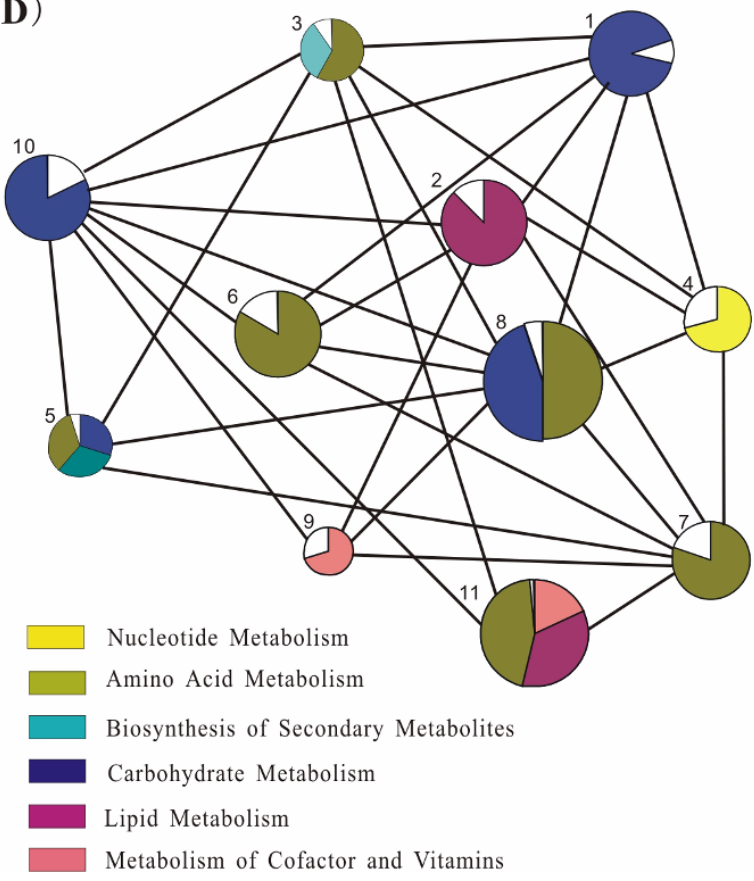

Figure 1 The Arabidopsis enzyme-enzyme metabolic network. (A) The interaction between two enzymes. Dots represent enzymes, directional edges represent metabolite flux. (B) Diagram showing in-degree, out-degree and bottlenecks. The in-degree of node $a$ is 5 whereas its out-degree is 1 , node $a$ and node $\beta$ are bottlenecks. The between-ness shown on the top left of each nodes. (C) The enzyme-enzyme network and the distribution of 173 WGD-enzymes. Dots represent enzymes and the lines represent the interactions among enzymes. Dots highlighted by red represent the WGDenzymes. (D) Cartography of Arabidopsis metabolic network. The circles represent modules (1 to 11) and the lines reflect the connections among modules. The area of color in each module is proportional to the number of enzymes that belong to the corresponding functional pathways. The blanks represent the enzymes whose corresponding pathways are not represented in the module. 
degree and out-degree only consider the partners connected directly to any particular node, whereas betweenness considers a node's position in the network. Usually nodes with relatively higher degrees are termed hubs (see example, node $\alpha$ in Figure 1B) and nodes with higher between-ness are named bottlenecks [18] (see examples, node $\alpha$ and node $\beta$ in Figure 1B).

In this study, we reconstructed the Arabidopsis metabolic network according to the recently updated Aracyc data (biochemical pathway database for Arabidopsis thaliana)[19]. By using graph theory for the analysis of the metabolic network, each enzyme was assigned with topological centralities (in-degree, out-degree and betweenness) to measure quantitatively its importance in the network. The observation that homeologs retained following WGD preferentially encode hub-bottleneck enzymes, provides us a first view of the relationship between metabolic networks and the retention of WGD-homeologs in Arabidopsis.

\section{Results}

\section{Hubs and bottlenecks tend to express highly and evolve} conservatively

The Arabidopsis enzyme-enzyme metabolic network was constructed using the recently updated Aracyc database [19] (see Methods). We retrieved 1785 directional interactions among 496 enzymes, of which 478 enzymes are included in a large network (Additional file 1) and the other 18 enzymes in 7 small clusters. The large connected network contains 1015 directional interactions and 379 bi-directional interactions (Figure 1C). In directional interaction, metabolite is the substrate or product of particular enzyme, whereas in bi-directional interaction, metabolite can be used as substrate as well as product by the same enzyme. The large connect network is highly modular and the 11 separated modules correspond to functional pathways (Figure 1D) (see Methods for modular analysis and Additional file 2 for description of 11 modules), confirming that the topological analysis of enzymes can reasonably reveal their functional status in the network. Topologically, the highly modular structure of the Arabidopsis metabolic network indicates the exis- tence of bottleneck enzymes, which tend to connect different modules/pathways. Between-ness (see Methods), which refers to the centrality of the considered enzymes in control of substrate-product fluxes in the network, was used to quantify this propensity.

A previous study [20] showed that genes encoding enzymes in the same pathway tend to co-express and core-metabolic pathways display tighter levels of transcriptional coordination, but the relationship between the mRNA transcription profiles of genes encoding enzymes and their importance in the metabolic network remains unknown. We did a correlation analysis between the expression parameters (maximum intensity, expression variation, see Methods and Additional file 3) and topological centralities (in-degree, out-degree, between-ness) of enzymes. Spearman correlation analysis showed that the in-degree, out-degree and between-ness positively correlate with expression intensity (Table 1). This indicates that genes encoding the hub and bottleneck enzymes tend to express with higher intensity.

We further explored whether genes with higher indegree, out-degree and between-ness evolve conservatively in orthologous gene pairs between Arabidopsis and Populus. The non-synonymous substitution rate, $\mathrm{Ka}$ was used to measure the evolutionary rate of the coding region (see Methods, Additional files 4 \&5). By correlating the average $K a$ of genes with their topological centralities, we found that the in/out-degree, out-degree and between-ness negatively correlate with the average $\mathrm{Ka}$. Also, the in-degree and between-ness negatively correlate with the average substitution rate of the 5 upstream and 3 ' downstream 1000-bp regions (Table 2). These results show that the genes encoding hub and bottleneck enzymes tend to be more conservative in their coding, 5 'upstream and 3'downstream regions. Taken together, genes encoding hubs and bottleneck enzymes tend to express highly and evolve conservatively.

\section{Hub and bottleneck enzymes prefer to retain homeologs through WGD}

The reconstructed Arabidopsis metabolic network enabled us to investigate the relationship between the

Table 1: The correlations between three topological centralities and the expression intensity, variation.

\begin{tabular}{|c|c|c|c|c|c|c|}
\hline & \multicolumn{2}{|c|}{$\begin{array}{l}\text { Max Intensity in developmental } \\
\text { stages }\end{array}$} & \multicolumn{2}{|c|}{$\begin{array}{c}\text { Max Intensity in shoot after } \\
\text { stress }\end{array}$} & \multicolumn{2}{|c|}{$\begin{array}{c}\text { Variation in developmental } \\
\text { stages }\end{array}$} \\
\hline & rho & Q value\# & rho & Q value & rho & $Q$ value \\
\hline In-degree & 0.155 & $3.96 \mathrm{E}-04^{* *}$ & 0.151 & $4.08 \mathrm{E}-04^{* *}$ & 0.026 & 0.11 \\
\hline Out-degree & 0.183 & $6.47 \mathrm{E}-05^{* *}$ & 0.173 & $1.19 \mathrm{E}-04^{* *}$ & 0.039 & 0.08 \\
\hline Betweenness & 0.130 & $1.81 \mathrm{E}-03^{* *}$ & 0.144 & $3.08 \mathrm{E}-04^{* *}$ & 0.005 & 0.16 \\
\hline
\end{tabular}

\#The q-values were obtained by the FDR correction of $p$ values from spearman correlation analysis, 2-tailed. 
Table 2: The correlations between three topological centralities and substitution rates in coding or 5' upstream, 3'downstream $1000 \mathrm{bp}$ regions.

\begin{tabular}{|c|c|c|c|c|c|c|}
\hline & \multicolumn{2}{|c|}{ Non-synonymous coding rate $(n=414)$} & \multicolumn{2}{|c|}{ 3' downstream 1000 bp region $(n=378)$} & \multicolumn{2}{|c|}{ 5' upstream 1000 bp region $(n=395)$} \\
\hline & rho & Q value\# & rho & Q value & rho & Q value \\
\hline In-degree & -0.201 & $6.47 \mathrm{E}-05^{* *}$ & -0.110 & $0.007^{* * *}$ & -0.161 & $5.93 \mathrm{E}-04^{* *}$ \\
\hline Out-degree & -0.177 & $6.47 \mathrm{E}-05^{* *}$ & -0.066 & $0.041^{*}$ & -0.082 & $0.026^{*}$ \\
\hline Betweenness & -0.155 & $5.93 \mathrm{E}-04^{* *}$ & -0.101 & $0.011^{*}$ & -0.138 & $0.002^{* *}$ \\
\hline
\end{tabular}

\#The q-values were obtained by the FDR correction of $\mathrm{p}$ values from spearman correlation analysis, 2-tailed.

retention of enzyme-homeologs through WGD and their centralities in the metabolic network. We identified enzymes as WGD-enzymes if these enzymes have at least one pair of homeologs, which were retained through the processes of gene gains and losses following WGD. The dataset of homeologs generated by WGD was retrieved from the Arabidopsis polyploidy database http:// Wolfe.gen.tcd.ie/athal/dup, and genes encoding 173 WGD-enzymes were identified (Additional file 6). Comparisons of in-degree, out-degree and between-ness distributions of WGD-enzymes with those of other enzymes show that the WGD-enzymes have significantly higher in in-degree, out-degree and between-ness scores (Figure 2A), indicating that the WGD-enzymes are preferentially located in hub and bottleneck positions of the network. In other words, genes encoding hub and bottleneck enzymes are preferentially retained as homeologs through WGD.

Previous research $[21,22]$ indicated that more ancient enzymes tend to have higher connectivity. So, we investigated whether the observed enrichment of WGDenzymes involved as hubs and bottlenecks in Arabidopsis metabolic network was due to their phylogenetic ages. Of the 173 WGD-enzymes in Arabidopsis, 162 were found to have at least one encoding-genes involved in ArabidopsisPopulus (Additional file 4) or Arabidopsis-rice ortholog groups (Additional file 5). Of the other 305 non-WGD enzymes, 281 were found to have at least one encoding genes involved in Arabidopsis-Populus or Arabidopsisrice ortholog groups. Chi-square test showed that WGDenzymes and non-WGD enzymes were not different in phylogenetic age $(162 / 173$ vs. $281 / 305,2$-sided, $p=0.59)$. Since the genome duplication event in Arabidopsis occurred 20-40 million years ago [2], significantly later than the split of Arabidopsis and Populus [5], the significant differences in connectivity (in-degree, out-degree and between-ness) among the metabolic enzymes must have already existed before the genome duplication event in Arabidopsis. The enrichment of WGD-enzymes in the hubs and bottlenecks in the metabolic network were not significantly influenced by the phylogenetic ages.
Plant genomes contain significant numbers of tandem duplicate genes. We also tested whether tandem duplicate genes tended to encode hub and bottleneck enzymes. The subset of tandem duplicates genes in Arabidopsis was retrieved from the TIGR database, http:// www.tigr.org/tdb/e2k1/ath1/TandemDups/Tandem-

Genes.html (criteria: e-value $<=1 \mathrm{e}-20$, only one unrelated gene was allowed to be interspersed within a cluster of tandem duplicated genes, 2500 genes were identified). Of the 478 metabolic network enzymes, we identified genes encoding 25 metabolic enzymes which retained tandem-homeologs (named by tandemenzymes, see Additional file 7). The comparison showed that the tandem-enzymes and the other enzymes have no significant difference in the in-degree, out-degree and between-ness (Additional file 8). So gene families encoding hub and bottleneck enzymes are not preferentially enlarged in their copy number by tandem duplication.

\section{WGD-enzymes tend to catalyze consecutive steps}

Random simulation was adopted to estimate the impact of preferential retention of WGD homeologs in hubs and bottlenecks. The identified 173 WGD-enzymes were used to retrieve the connected WGD-enzymes, and 255 interactions (named by interaction between WGDenzymes, IBWE) were obtained (Figure 2B). Simulation analysis involved two steps, (a) the 173 enzymes were randomly assigned in the network, (b) edges connecting two selected enzymes were marked and the number of edges $k$ was recorded. Then steps from (a) to (b) were repeated 10000 times, and resulted in a normal distribution $N(182,22)$ of $k$ (Figure $2 \mathrm{C}$ ). The observed number of 255 interactions obtained from the WGD-enzymes significantly deviated from random expectation (Z-score $=$ $(255-183) / 22=3.27, \mathrm{p}<0.01)$. This indicated that WGDenzymes tend to be connected, revealing that they tend to catalyze consecutive steps in the pathways (see example in Figure 3).

The simulation analysis indicated the retention of genes encoding enzyme-homeologs was not independent of the retention of the genes encoding their interacting enzymehomeologs. For a metabolic interaction $E_{1}->E_{2}$, after the 
A)

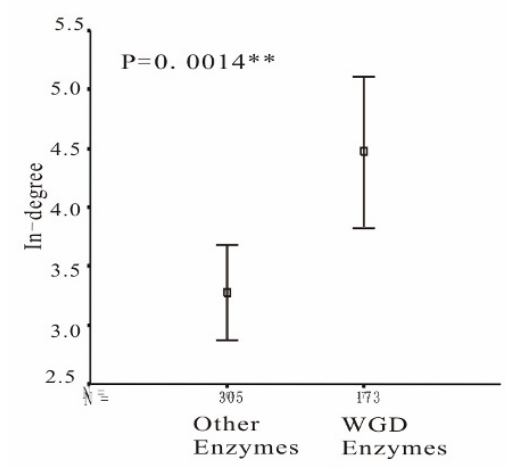

B)

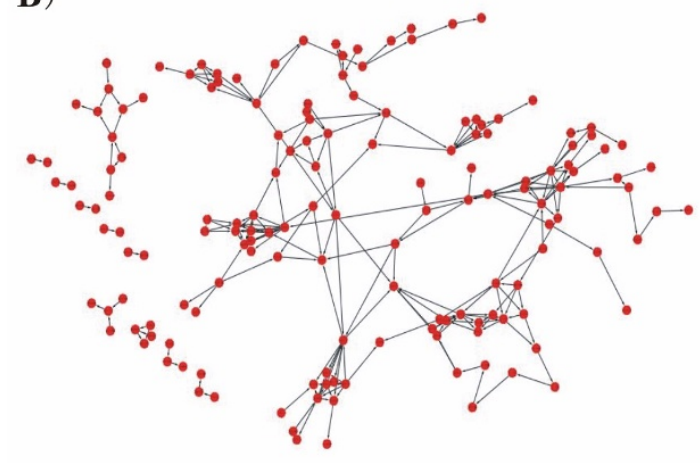

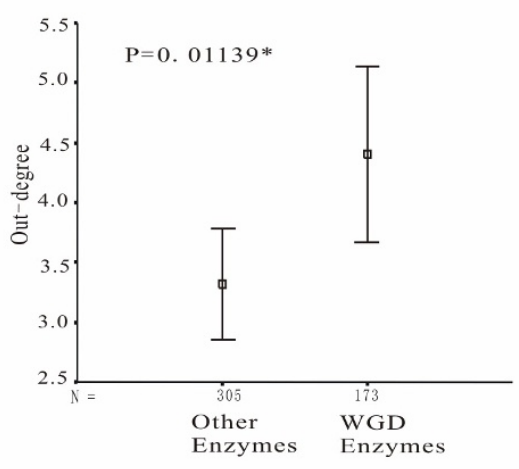

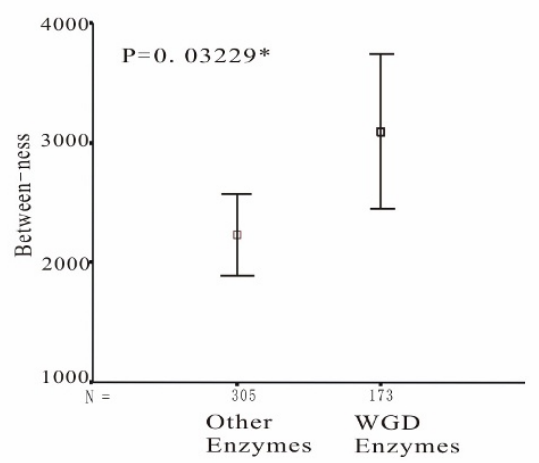

C)

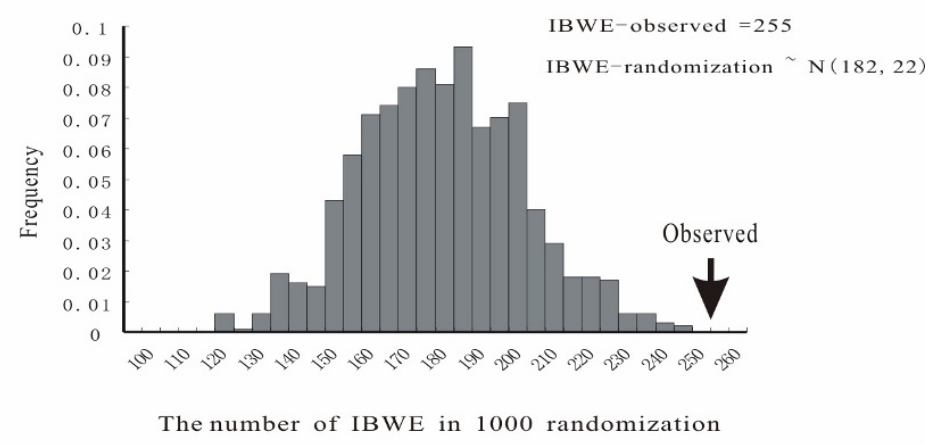

Figure 2 Analysis of WGD-enzymes in metabolic network. (A) Comparison of three topological centralities between WGD-enzymes and the other enzymes. (B) Sub-network of WGD-enzymes. Dots represent enzymes and the lines represent the interactions among enzymes. (C) The distribution of IBWEs in 1000 randomization simulations. The arrow on the right hand side represents the observed number of IBWE.

whole genome duplication, the retention of $E_{1}$ could influenced by the retention of $E_{2}$, or vice versa. Obviously, some interactions promote the simultaneous retention of connected enzymes and could significantly increase the number of IBWE. The number of this type of interactions followed a normal distribution because $255-N(182,22)=$ $N(72,22)$ (see Figure $2 C$ ). To investigate how many WGDenzymes are due to this type of interaction, we first (a), removed the information of WGD-enzymes in the network. Then a number, $m$, were drawn from a normal distribution $N(72,22)$. (b) By randomly assigning $m$ edges to the network, nodes connected by the selected edges were marked, and then the number of nodes, $f$, was recorded. Repeating two steps from (a) to (b) 10000 times, $f$ followed a normal distribution as $N(62,16)$, This indicates that this type of interactions render about 62 enzymes to be WGD-enzymes with the standard error of 16 . In total, approximately one-third (62/173) of the WGD-enzymes were attributable to the impact of metabolic interaction.

We applied comparative genomics to explore whether the interactions connected by WGD enzymes in Arabi- dopsis were also likely connected by WGD enzymes in Populus. The datasets of Populus enzymes from http:// genome.jgi-psf.org/Poptr1 1/, and the Populus WGDhomeologs were retrieved from http:// chibba.agtec.uga.edu/duplication/[23]. Our analysis identified Populus genes encoding 226 enzymes which retain at least one pair of WGD-homeologs (Additional file 9). Among the 1394 Arabidopsis enzyme-enzyme interactions, 255 interactions were identified to be connected by Arabidopsis WGD enzymes (IBWE, Figure 2B), while 68 ortholog interactions (the interactions between orthologs in both Arabidopsis and Populus) were found to be connected by WGD-enzymes in Populus. Of the 255 Arabidopsis IBWE, 32 ortholog interactions also are connected by WGD-enzymes in Populus. (see the example, Figure 3$)$. The propensity $(12 \%, 32 / 255)$ is significantly higher than the expected value $(5 \%, 68 / 1394)(p<$ 0.01 , two-tailed, chi-square test), indicating that the interactions connected by WGD-enzymes in Arabidopsis indeed also tend to be connected by WGD-enzymes in Populus. 


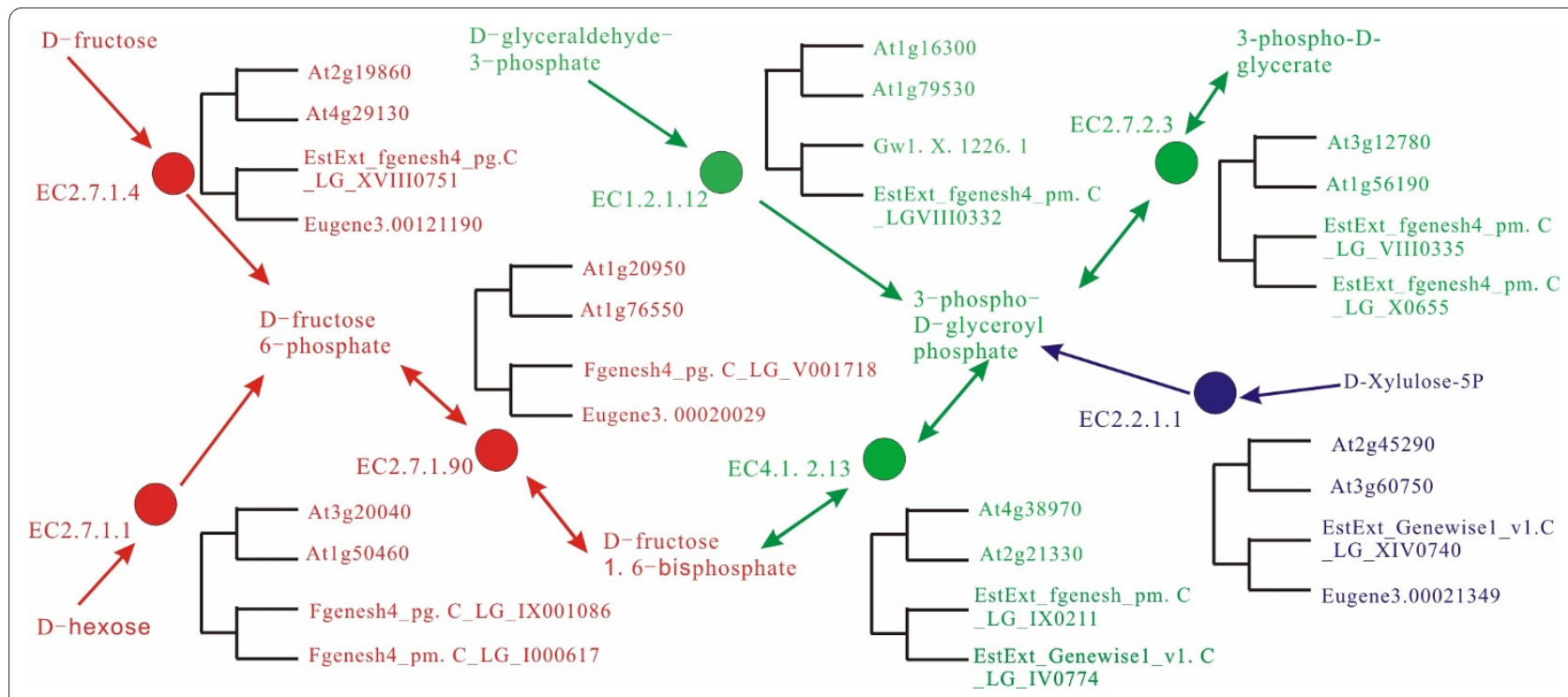

Figure 3 A sub-network contains seven Arabidopsis/Populus WGD-enzymes which locate in the three inter-connected pathways. The fructose metabolism, gluconeogenesis and the pentose phosphate pathways were marked by red, green blue fonts, respectively.

Figure 3 demonstrated a sub-network containing seven enzymes from the 32 IBWEs in both Arabidopsis and Populus. The seven enzymes locate in the inter-connected positions of three pathways, the fructose metabolism, gluconeogenesis and the pentose phosphate pathways. Phylogenetic analysis revealed that the seven genes were preferentially retained as parallel paralogs in the process of WGD after the two species split (see Methods). Genes encoding these important enzymes were simultaneously duplicated in the process of WGD, hence metabolic flux has simultaneously increased, maintaining the balance of the metabolite flux.

Finally, in the process of WGD, genes encoding hub and bottleneck enzyme can be easily retained their homeologs, providing not only one extra copy of individual enzymes but also another set of interconnected enzymes for the consecutive steps in the pathways.

\section{Discussion}

The gain/loss of homeologs was an important event in the evolution of the plant genome. Previous analysis in Arabidopsis [11] showed that some chromosome islands of retention contain 'connected genes' following genome duplication. Those genes are mainly from families encoding components of the proteasome/protein modification complexes, signal transduction machinery, ribosomes and transcription factor complexes. Our analysis indicated that genes encoding hub and bottleneck enzymes in the Arabidopsis metabolic network tend to be highly expressed and more conserved. This results support the previous observations that highly expressed genes evolve slowly in yeast [8] and genes that have a lower propensity to be lost in the evolution accumulate fewer substitutions in their protein sequences and tend to be essential for the organism viability, tend to be highly expressed, and have many interacted proteins based on the analysis of the vertebrate genomes [24].

Our further analysis revealed that genes encoding hub and bottleneck enzymes in the Arabidopsis metabolic network tend to preferentially retain homeologs after WGD and the simultaneous retention of WGD-homeologs encoding enzymes which catalyze consecutive steps in a pathway is highly favored. This phenomena can be best explained by the dosage-sensitive relationship in the gene balance hypothesis which has been demonstrated in yeast and humans [25], maize and Drosophila [26] and also by the theoretical prediction [27]. In brief, this hypothesis presumes that after long term evolution, "connected genes" of multisubunit complexes in the present genomes have been in an optimum balance status and changes of the individual genes in the subunit would display dosage sensitivity, resulting in out-of-balance phenotypes which have disadvantages in fitness in the evolution [28-30]. In the Arabidopsis metabolic network, genes encoding hub enzymes are in the important positions and usually connected with many other enzymes, forming a sub-network, and are very likely to be more sensitive to the dosage effects and indeed preferentially retained after WGD.

The simulation analysis revealed that about one-third of the WGD-enzymes were attributable to the impact of metabolic interaction, the comparative genomics analysis demonstrated that $12 \%$ of interactions connected by WGD-enzymes in Arabidopsis are connected by WGDenzymes in Populus. The results indicate that genes involving in this type of subnetwork-subnetwork connec- 
tions tend to form an large evolutionary unit, requiring simultaneous retention of interconnected genes. We assume that the metabolism intermediates between the upstream and downstream enzymes are the key biological driving force. Maintaining balanced metabolic flux is important for the survival of plants. Recent experimental data [31] demonstrated that over-expression of a gene in the OSC-Cyp 708 operon-like gene cluster resulted in the abnormal development of the Arabidopsis plant. Clear dosage effects were observed in the genetic analysis of saponin biosynthesis pathway in oats [32]. Sad1 encodes 2,3 -oxidosqualene to produce $\beta$-Amyrin, which eventually is used to produce avenacin A-1 by Sad3 and Sad4. Double mutant analysis revealed that Sad1Sad1sad3sad3, Sad1Sad1-sad4sad4 and Sad1sad1-sad3sad3 have abnormal root development, while Sad1sad1sad4sad4 has the normal root development. In many cases, accumulation of metabolic intermediates would be toxic to plants and simultaneous duplication of consecutive steps in the metabolic pathways is required and favoured.

Apart from the potential dosage sensitivity of metabolic interaction, simultaneous expression divergence (subfunctionalization, DDC model [30]) of both interacting enzymes-homeologs could also promote the retention of homeologs, in which the coordinated expressional divergence has the strongest effect in achieving the simultaneous retention of enzyme-homeologs. Four interacting enzyme pairs showed concerted divergence in transcript expression of developmental stages (Additional file 10). That is, in the glycerophospholipid metabolism pathway, the interacting enzymes, EC4.1.1.65 and EC3.1.4.4, were found to be retained as WGD-paralogs, the retained paralogs of At1g52570-At5g57190 and At3g15730At4g25970 have both coordinately diverged and express in different developmental stages for the benefit of the interaction. The other three divergently connected enzymes showing co-expression are EC2.3.1.12 and EC1.8.1.4, which were found in the gluconeogenesis pathway, EC2.3.16 and EC2.3.3.1, which mediate fatty acid metabolism and the citrate cycle, and EC2.6.1.2 and EC2.3.1.1 which mediate glutamate metabolism and metabolism of amine groups. The concerted divergence of WGD-enzymes may provide an easy route for the retention of consecutive steps. Since only four such interacting enzyme pairs were found, this mechanism seems to make only a small contribution to the retention of enzyme-homeologs.

The gene balance hypothesis also predicts that more "connected" genes are less likely to be retained as a tandem or transposed duplicate and are more likely to be retained postpaleotetraploidy [30]. It is indeed that genes encoding the hub and bottleneck enzymes in the Arabidopsis metabolic network prefer to retain homeologs through WGD but are not preferentially enlarged in their copy number by tandem duplication.

Arabidopsis is a good model for plant polyploidization studies. Many economically important plants such as cotton, Brassica rapa, potato, soybean, maize and wheat are polyploids. Through millennia of hybridization and domestication, wheat cultivars that are diploid, tetraploid and hexaploid have been produced. After the divergence from the ancestral sorghum genome, the tetra-ploidization of the progenitor genomes of maize occurred about $5 \sim 12$ million years ago [33]. Polyploidization eventually leads to offspring that are distinguished from its progenitors. Analysis of the Arabidopsis metabolic network shows that both its robustness has been enhanced by the process of WGD. We predicted that the polyploidizations of these main agricultural plants would have increased their environmental adaptability and provided humanneeded traits for domestication. Hence, polyploid breeding might be worth more attention in plant breeding programs. Also in transgenic-based plant breeding, the simultaneous engineering of a set of hub and bottleneck genes/enzymes would be a better strategy than manipulation of a single gene/enzyme. As more plant genomes are sequenced, a deeper view of the evolutionary impact of WGD can help us to develop better breeding strategies in modern agriculture.

\section{Conclusions}

In this study, we analyzed the Arabidopsis metabolic network by assigning the enzymes with three topological measures, in-degree, out-degree and the between-ness. Comprehensive analyses were carried out between the three centralities and the characteristics of the encoded genes, such as expression intensity, evolutionary rate, and retention of homeologs through WGD. Our results revealed that genes encoding hub and bottleneck enzymes in the metabolic network are preferentially retained after WGD. Furthermore the finding suggested that the retention of metabolic genes was influenced by their interactions and validated that the preferential retention of WGD homeologs encoding hub and bottleneck enzymes is due mainly to the potential dosage effect among interacted genes encoding enzymes if exist). Our results could help us get a deeper view of the evolution of plant metabolic network.

\section{Methods}

\section{Construction of Arabidopsis metabolic network}

Three files were downloaded to reconstruct the Arabidopsis metabolic network: (a) an expert-curated list of Arabidopsis encoded enzymes and the corresponding genes from Aracyc (ftp://ftp.plantcyc.org/Pathways, june,2008 updated) [19], (b) a "reaction" file ftp://ftp. genome.jp/pub/kegg/ligand/reaction/reaction/ to scan all 
catalyzed reactions of Arabidopsis enzymes [34], (c) a "reaction_mapformula.lst" file ftp://ftp.genome.jp/pub/ kegg/ligand/reaction/reaction mapformula.lst to obtain the information of metabolites in reactions. The reactions between metabolites were used to determine the interactions among enzymes. In the Figure 1A, enzyme EC2.7.7.9 uses alpha-D-glucose-1-phosphate as substrate to produce UDP-glucose, which is then used by enzyme EC5.1.3.2, the interaction was defined as EC2.7.7.9 T EC 5.1.3.2. Because small molecules, $\mathrm{H}+\mathrm{NADH}, \mathrm{NADP}$, NADPH, NH3, ATP, ADP, AMP, NAD, CoA, O2, CO2, Glu and pyrophosphate, are involved in many reactions or are used as carriers for transferring electrons, they were excluded from the analysis $[35,36]$.

\section{Calculation of node in-degree, out-degree and between- ness}

The in-degree was calculated by the number of enzymes providing substrates for the considered enzyme, whereas the out-degree was calculated by the number of enzymes using the products of the considered enzyme as substrates. The between-ness was calculated by the "breathfirst tree" based algorithm as following steps [18,37]. (a) The calculation was initialized by defining the betweenness of every vertex $j$ in the network as $B(j)=0$. (b) Starting from vertex $i$, a breadth-first tree http://en.wikipedia.org/wiki/Breadth-first search was built with $i$ on the top, those that were nearest to $i$ directly below and those that were farthest from $i$ at the bottom. Each node was placed at a certain level of the tree based on its shortest metabolic reaction step. (c) $P(n)=1$ was assigned to every vertex $j$ in the tree. For every vertex $j P(j)=\sum_{1}^{k} P(k)$, where $k$ is the set of nodes that directly connect ("provides substrates") to $j$. (d) $B(j)=1$ was assigned to every vertex $j$ in the tree. (e) Starting from bottom vertex $j$ of the tree, $B(j)$ was added to the corresponding variable of the predecessor of $j$. If $j$ had more than one predecessor ("enzyme $k$ provides more than one substrate to the enzyme $j ")$, each predecessor $k$ was assigned the value of $\frac{P(k)}{P(i)} B(j)$. (f) Step $(e)$ was performed for every vertex in the tree. (g) Steps $(b)-(f)$ were repeated for every vertex in the network. Finally, every vertex in the network was assigned with a between-ness value which is the sum of its between-ness of every sub-tree involved.

\section{Modular analysis of the metabolic network}

The node distributions of $\mathrm{P}<\mathrm{In}>$ and $\mathrm{P}<\mathrm{Out}>$ were used to investigate the frequency of the in-degree and outdegree. The least-squares method was used to estimate power-law exponent of $p(k) \infty \boldsymbol{k}^{-t}$ for log-transformed data (t, power exponent; $k$, in/out-degree). Since the estimated power-law exponent was 1.67 , methods for study of scalefree structure was applied in analysis the Arabidopsis metabolic network. The algorithm of Guimera and Amaral [37], with parameter settings as iteration factor $=1.0$, cooling factor $=0.95$ and number of randomization $=$ 100 , was used to measure the extent of modularity of network and separate the network into topological modules. The Kobas toolkit [38] was used to infer the frequent pathways in every topologically separated module.

\section{Analysis of transcription datasets of Arabidopsis}

The gene transcription datasets of different developmental stages were obtained from the Affymetrix ATH1 data (TAIR accession number, ME00319) [39]. The raw data were normalized by the Affymetrix detection algorithms in the MAS5 library, the background levels and PM/MM ratios were corrected according to the Affymetrix Statistical Algorithms. Based the estimated expression values of probes, the expression values of corresponding 22,380 Arabidopsis genes. After filtering the mixture of RNA pools or measurement of the same developmental stages, 59 datasets of ME00319, which measured the developmental stages of Arabidopsis, were selected for downstream analysis (Additional file 3). Expression intensities were averaged among three replicates for every developmental stage.

The Expression Variation index $V_{i}$ was used to measure the variations of gene i in expressional level across developmental stages [40].

$$
V_{i}=\frac{\sum_{j=1}^{n}\left(1-\frac{\log S_{i j}}{\log S_{(i, \max )}}\right)}{n-1}
$$

where $\mathrm{n}$ is the number of stages, $S_{i j}$ is the expression signal of gene i in tissue $\mathrm{j}, S_{(i, \max )}$ is the highest expression signal of gene i across the stages, if the $S_{i j}$ is lower than 50 we arbitrary let it be 50 to minimize the influence of noise from low intensities. The $V_{i}$ value ranges form 0 to 1 , higher value indicating higher variations in expressional level across stages or tend to be stage specific genes.

The expression maximum density of enzymes were calculated by $M_{\max }=\frac{1}{n} \sum_{1}^{n} S_{(i, \max )}$ (where $n$ was the number of genes annotated with this enzyme, $S_{(i, \max )}$ was the maximum expression density of gene $i$ among the developmental stages). The expression variation of enzymes were calculated by $V=\frac{1}{n} \sum_{1}^{n} v_{(i)}$ (where $n$ was the number of genes annotated with this enzyme, $v_{(i)}$ was Expression Variation index gene $i$ ). 
The Pearson correlation coefficient $\left(P_{i j}\right)$ was used to measure the co-expression between gene $i$ and gene $j$ as following formula:

$$
P_{i j}=\frac{\sum_{k=1}^{n}\left(S_{k i}-\bar{S}_{i}\right)\left(S_{k j}-\bar{S}_{j}\right)}{\sqrt{\sum_{k=1}^{n}\left(S_{k i}-\bar{S}_{i}\right)^{2}} \sqrt{\sum_{k=1}^{n}\left(S_{k j}-\bar{S}_{j}\right)^{2}}}
$$

where $\mathrm{n}=$ the number of developmental stages, $S_{k i}$ and $S_{k j}$ were the expression value of gene $i$ and $j$ under condition $k ; \bar{S}_{i}$ and $\bar{S}_{j}$ were the mean expression value of gene $i$ and $j$ across the stages, the $P_{i j}$ was between -1 and 1 , with 1 standing for highly co-expressed whereas -1 standing for highly divergent expressed.

The datasets, which time-seriously measured the shoot tissues responding to various abiotic stress (exogenous factors: cold, genotixic, osmotic, salt, UV-B, wound, drought, and heat, see Table S3), were also analyzed with the same procedures.

\section{Identification of ortholog groups between Arabidopsis and Populus}

The phylogenetic tree approach was used to infer orthologs between Arabidopsis and Populus. The proteomes of Arabidopsis thaliana, Populus trichocarpa and Oryza sativa japonica were downloaded from http:// www.tigr.org/tdb/e2k1/ath1/, http://genome.jgi-psf.org/ Poptr1 1/ and http://rice.plantbiology.msu.edu/ index.shtml. The sequences of three species were used an all-against-all BLAST with an cutoff of $1 \mathrm{e}-10$, by transforming the E-values with the absolute values of their logarithm[41], the score matrix were constructed and used for similarity clustering with Markov Clustering [42]. The protein clusters containing the Arabidopsis metabolic genes were used for phylogenetic analysis.

The protein sequences of members in each cluster were aligned with ClustalW [43] and the alignments were used to generate neighbor-joining trees with the two-parameter substitution correction. The phylogenetic trees were rooted at midpoints. By reconciling between phylogentic tree and the species tree ((Arabidopsis thaliana, Populus trichocarpa), Oryza sativa japonica)) with Notung [44], the ortholog groups were identified between Arabidopsis and Populus.

\section{Measurement of the evolutionary rates in coding region and 5' upstream, 3' downstream regions of genes encoding enzymes in Arabidopsis}

The Clustalw was used to globally align two amino acid sequences of orthologs between Arabidopsis and Populus, and the corresponding coding sequences were realigned with the gaps in the alignment trimmed. The $K a$ was estimated from the codon-based nucleotide sequence alignment by using the Yang-Nielsen maximum-likelihood method implemented in the yn00 program of the PAML package[45]. To calculate the substitution rates in Arabidopsis genes 5' 1000bp upstream and 3' 1000 bp downstream region were calculated against that of Populus orthologs. The Clustalw software [43] were used to globally align the non-coding regions of orthologs, the substitution rate per sites $K_{5 u}$ and $K_{3 u}$ with the Kimura two-parameter model were calculated by dismat program of the EMBOSS package [46]. For a Arabidopsis gene $i$ with more than one orthologs in Populus, the smallest of calculated $K a, K_{5 u}$ and $K_{3 u}$ were selected as $K a_{(i)}, K_{5 u(i)}$ and $K_{3 u(i)}$.

The average divergence in coding regions, 5 'upstream and 3'downstream region of enzyme could be calculated by the formula $D_{\text {coding }}=\frac{1}{n} \sum_{i=1}^{n} K a_{(i)}, \quad D_{5 u}=\frac{1}{n} \sum_{1}^{n} K_{5 u(i)}$ and $D_{3 u}=\frac{1}{n} \sum_{1}^{n} K_{3 u(i)}$, the $n$ was the number of genes annotated by this enzyme, $K a_{(i)}, K_{5 u(i)}$ and $K_{3 u(i)}$ was the substitution rate in coding, upstream and downstream non-coding regions of Arabidopsis gene.

\section{Statistical analysis and computational methods}

Spearman's rank correlation coefficients were estimated to evaluate the correlations between three topological centralities and expression intensities, expression variation, substitution rates of coding regions of gene encoding enzymes. The p-values were FDR-corrected by using the $\mathrm{Q}$-value program in $\mathrm{R}$ package [47]. The comparison of topological centralities between WGD-enzymes and the other enzymes were done by using Manny-Whitney $\mathrm{U}$ with two-tail test. Computations were performed on a Linux cluster with 16 nodes (Intel 5130, 2.0 GHz CPU, 4G memory, Research Center for Systematic and Evolutionary Botany, Institute of Botany, CAS). Perl http://perl.org and $\mathrm{R}$ http://www.r-project.org/ scripts were used for analysis, and can be obtained on request.

\section{Additional material}

Additional file 1 Table S1. 478 enzymes in the largest network and their topological centralities.

Additional file 2 Table S2. 11 topological modules and their major functions.

Additional file $\mathbf{3}$ Table S3. The description of Arabidopsis microarry datasets.

Additional file 4 Table S4. The identified ortholog groups between Arabidopsis and Populus by phylogenetic trees.

Additional file $\mathbf{5}$ Table S5. The identified ortholog groups between Arabidopsis and rice by phylogenetic trees. 
Additional file 6 Table S6. 173 Arabidopsis WGD-enzymes and their coding homeologs.

Additional file 7 Table S7. 25 Arabidopsis tandem-enzymes and their coding tandem homeologs in the Arabidopsis metabolic network.

Additional file 8 Figure S1. Comparison of three topological centralities between enzymes retaining tandem-homeologs and the other enzymes.

Additional file 9 Table S8. 226 Populus WGD-enzymes and their coding homeologs.

Additional file 10 Figure S2. The expression profiles of four connected enzyme-homeologs in the 59 developmental stages.

\section{Abbreviations}

Ka: non-synonymous substitution rates of coding region; $\mathrm{K} 5 \mathrm{u}$ : substitution rate of 5'UTR; K3u: substitution rate of 3'UTR; WGD: whole genome duplication; WGD: enzyme, the enzyme retaining paralogs through whole genome duplication; IBWE: the interaction between WGD-enzymes.

\section{Authors' contributions}

$\mathrm{XW}$ and $\mathrm{XQ}$ performed the experiments and analyzed the data, XQ directed the project and contributed the materials and analysis tools. XW and XQ wrote the paper. All authors read and approved the final manuscript.

\section{Acknowledgements}

We thank Cathie Martin, Anne Osbourn and Catherine Goodman for a critical reading and discussions. This work was supported by the National Basic Research Program of China (2007CB108800) and Chinese Academy of Sciences (grants number KSCX2-YW-N-028 and A Hundred Talents Program).

\section{Author Details}

Key Laboratory of Photosynthesis and Environmental Molecular Physiology, the Institute of Botany, Chinese Academy of Sciences, Beijing 100093, China

Received: 12 January 2010 Accepted: 18 May 2010

Published: 18 May 2010

\section{References}

1. Bowers JE, Chapman BA, Rong J, Paterson AH: Unravelling angiosperm genome evolution by phylogenetic analysis of chromosomal duplication events. Nature 2003, 422(6930):433-438.

2. Blanc $\mathrm{G}$, Hokamp K, Wolfe $\mathrm{KH}$ : A recent polyploidy superimposed on older large-scale duplications in the Arabidopsis genome. Genome Res 2003, 13(2):137-144.

3. Paterson AH, Bowers JE, Chapman BA: Ancient polyploidization predating divergence of the cereals, and its consequences for comparative genomics. Proc Natl Acad Sci USA 2004, 101(26):9903-9908.

4. Wang $X$, Shi $X$, Hao B, Ge S, Luo J: Duplication and DNA segmental loss in the rice genome: implications for diploidization. New Phytol 2005 165(3):937-946

5. Tuskan GA, Difazio S, Jansson S, Bohlmann J, Grigoriev I, Hellsten U, Putnam N, Ralph S, Rombauts S, Salamov A, et al:: The genome of black cottonwood, Populus trichocarpa (Torr. \& Gray). Science 2006, 313(5793):1596-1604.

6. Blanc G, Wolfe KH: Functional divergence of duplicated genes formed by polyploidy during Arabidopsis evolution. Plant Cell 2004, 16(7):1679-1691.

7. Subramanian S, Kumar S: Gene expression intensity shapes evolutionary rates of the proteins encoded by the vertebrate genome. Genetics 2004, 168(1):373-381

8. Pal C, Papp B, Hurst LD: Highly expressed genes in yeast evolve slowly. Genetics 2001, 158(2):927-931.

9. Fraser HB, Wall DP, Hirsh AE: A simple dependence between protein evolution rate and the number of protein-protein interactions. $B M C$ Evol Biol 2003, 3:11.

10. Blomme T, Vandepoele K, De Bodt S, Simillion C, Maere S, Peer Y Van de: The gain and loss of genes during 600 million years of vertebrate evolution. Genome Biol 2006, 7(5):R43

11. Thomas BC, Pedersen B, Freeling M: Following tetraploidy in an Arabidopsis ancestor, genes were removed preferentially from one homeolog leaving clusters enriched in dose-sensitive genes. Genome Res 2006, 16(7):934-946.
12. Gout JF, Duret L, Kahn D: Differential retention of metabolic genes following whole-genome duplication. Mol Biol Evol 2009, 26(5):1067-1072.

13. Dixon RA: Natural products and plant disease resistance. Nature 2001, 411(6839):843-847.

14. Qi X, Bakht S, Leggett M, Maxwell C, Melton R, Osbourn A: A gene cluster for secondary metabolism in oat: implications for the evolution of metabolic diversity in plants. Proc Natl Acad Sci USA 2004, 101(21):8233-8238

15. Qi X, Bakht S, Qin B, Leggett M, Hemmings A, Mellon F, Eagles J, WerckReichhart $D$, Schaller $H$, Lesot $A$, et al: $A$ different function for a member of an ancient and highly conserved cytochrome P450 family: from essential sterols to plant defense. Proc Natl Acad Sci USA 2006, 103(49):18848-18853.

16. Barabasi AL, Oltvai ZN: Network biology: understanding the cell's functional organization. Nat Rev Genet 2004, 5(2):101-113.

17. Sweetlove LJ, Fell D, Fernie AR: Getting to grips with the plant metabolic network. Biochem J 2008, 409(1):27-41.

18. Yu H, Kim PM, Sprecher E, Trifonov V, Gerstein M: The importance of bottlenecks in protein networks: correlation with gene essentiality and expression dynamics. PLoS Comput Biol 2007, 3(4):e59.

19. Mueller LA, Zhang P, Rhee SY: AraCyc: a biochemical pathway database for Arabidopsis. Plant Physiol 2003, 132(2):453-460.

20. Wei H, Persson S, Mehta T, Srinivasasainagendra V, Chen L, Page GP, Somerville C, Loraine A: Transcriptional coordination of the metabolic network in Arabidopsis. Plant Physiol 2006, 142(2):762-774.

21. Papp B, Pal C, Hurst LD: Metabolic network analysis of the causes and evolution of enzyme dispensability in yeast. Nature 2004 429(6992):661-664.

22. Light $\mathrm{S}$, Kraulis $P$, Elofsson A: Preferential attachment in the evolution of metabolic networks. BMC Genomics 2005, 6:159.

23. Tang $H$, Bowers JE, Wang $X$, Ming R, Alam M, Paterson AH: Synteny and collinearity in plant genomes. Science 2008, 320(5875):486-488.

24. Krylov DM, Wolf YI, Rogozin IB, Koonin EV: Gene loss, protein sequence divergence, gene dispensability, expression level, and interactivity are correlated in eukaryotic evolution. Genome Res 2003, 13(10):2229-2235.

25. Papp B, Pal C, Hurst LD: Dosage sensitivity and the evolution of gene families in yeast. Nature 2003, 424(6945):194-197.

26. Birchler JA, Bhadra U, Bhadra MP, Auger DL: Dosage-dependent gene regulation in multicellular eukaryotes: implications for dosage compensation, aneuploid syndromes, and quantitative traits. Dev Biol 2001, 234(2):275-288.

27. Veitia RA: Rosetta Stone proteins: "chance and necessity"? Genome Biol 2002, 3(2):. INTERACTIONS1001

28. Birchler JA, Veitia RA: The gene balance hypothesis: from classical genetics to modern genomics. Plant Cell 2007, 19(2):395-402.

29. Freeling M, Thomas BC: Gene-balanced duplications, like tetraploidy, provide predictable drive to increase morphological complexity. Genome Res 2006, 16(7):805-814.

30. Freeling M: Bias in plant gene content following different sorts of duplication: tandem, whole-genome, segmental, or by transposition. Annu Rev Plant Biol 2009, 60:433-453.

31. Field B, Osbourn AE: Metabolic diversification--independent assembly of operon-like gene clusters in different plants. Science 2008, 320(5875):543-547.

32. Mylona P, Owatworakit A, Papadopoulou K, Jenner H, Qin B, Findlay K, Hill L, Qi X, Bakht S, Melton R, Osbourn A: Sad3 and sad4 are required for saponin biosynthesis and root development in oat. Plant Cell 2008, 20(1):201-212

33. Schnable PS, Ware D, Fulton RS, Stein JC, Wei F, Pasternak S, Liang C, Zhang J, Fulton L, Graves TA, et al:: The B73 maize genome: complexity, diversity, and dynamics. Science 2009, 326(5956):1112-1115.

34. Kanehisa M, Goto S: KEGG: kyoto encyclopedia of genes and genomes. Nucleic Acids Res 2000, 28(1):27-30

35. Ma H, Zeng AP: Reconstruction of metabolic networks from genome data and analysis of their global structure for various organisms. Bioinformatics 2003, 19(2):270-277.

36. Huss M, Holme P: Currency and commodity metabolites: their identification and relation to the modularity of metabolic networks. IET Syst Bio/ 2007, 1(5):280-285

37. Girvan M, Newman ME: Community structure in social and biological networks. Proc Natl Acad Sci USA 2002, 99(12):7821-7826. 
38. Wu J, Mao X, Cai T, Luo J, Wei L: KOBAS server: a web-based platform for automated annotation and pathway identification. Nucleic Acids Res 2006:W720-724.

39. Schmid M, Davison TS, Henz SR, Pape UJ, Demar M, Vingron M, Scholkopf B, Weigel D, Lohmann JU: A gene expression map of Arabidopsis thaliana development. Nat Genet 2005, 37(5):501-506.

40. Yanai I, Benjamin H, Shmoish M, Chalifa-Caspi V, Shklar M, Ophir R, BarEven A, Horn-Saban S, Safran M, Domany E, et al:: Genome-wide midrange transcription profiles reveal expression level relationships in human tissue specification. Bioinformatics 2005, 21(5):650-659.

41. Shiu SH, Shih MC, Li WH: Transcription factor families have much higher expansion rates in plants than in animals. Plant Physiol 2005, 139(1):18-26.

42. Van Dongen S: Graph clustering by flow simulation. $\ln P h D$ thesis University of Utrecht, Utrecht, The, Netherlands; 2000.

43. Thompson JD, Gibson TJ, Higgins DG: Multiple sequence alignment using ClustalW and ClustalX. Curr Protoc Bioinformatics 2002, Chapter 2(3):

44. Chen K, Durand D, Farach-Colton M: NOTUNG: a program for dating gene duplications and optimizing gene family trees. $J$ Comput Biol 2000, 7(3-4):429-447.

45. Yang Z: PAML: a program package for phylogenetic analysis by maximum likelihood. Comput Appl Biosci 1997, 13(5):555-556.

46. Rice P, Longden I, Bleasby A: EMBOSS: the European Molecular Biology Open Software Suite. Trends Genet 2000, 16(6):276-277.

47. Storey JD, Tibshirani R: Statistical significance for genomewide studies. Proc Natl Acad Sci USA 2003, 100(16):9440-9445.

doi: 10.1186/1471-2148-10-145

Cite this article as: Wu and Qi, Genes encoding hub and bottleneck enzymes of the Arabidopsis metabolic network preferentially retain homeologs through whole genome duplication BMC Evolutionary Biology 2010, 10:145

Submit your next manuscript to BioMed Central and take full advantage of:

- Convenient online submission

- Thorough peer review

- No space constraints or color figure charges

- Immediate publication on acceptance

- Inclusion in PubMed, CAS, Scopus and Google Scholar

- Research which is freely available for redistribution

Submit your manuscript at www.biomedcentral.com/submit
Ciomed Central 